\title{
miR-196b/miR-1290 participate in the antitumor effect of resveratrol via regulation of IGFBP3 expression in acute lymphoblastic leukemia
}

\author{
WEI ZHOU, SHUNQING WANG, YI YING, RUIQING ZHOU and PING MAO \\ Department of Hematology, Guangzhou First People's Hospital, Guangzhou Medical University, \\ Guangzhou, Guangdong 510180, P.R. China
}

Received June 29, 2016; Accepted August 13, 2016

DOI: $10.3892 /$ or.2016.5321

\begin{abstract}
MicroRNAs play critical roles in the progression of acute lymphoblastic leukemia (ALL). Previous studies have indicated that miR-196b and miR-1290 play critical roles in T-cell ALL (T-ALL) and B-cell ALL (B-ALL), respectively. Resveratrol, a natural edible polyphenolic phytoalexin, possesses certain anticancer activities. Nevertheless, the mechanism involved in the regulation of ALL by resveratrol is still poorly understood. The present study aimed to reveal the potential mechanism underlying the antitumor effect of resveratrol in ALL focusing on miRNAs. Research indicates that insulin-like growth factor binding protein 3 (IGFBP3) plays a critical role in the aetiology of ALL. In the present study, we first demonstrated that the expression of IGFBP3 was decreased in ALL patients. We further identified that miR196b and miR-1290 were overexpressed in T-ALL TALL-104 and B-ALL SUP-B15 cell lines, respectively. Moreover, resveratrol markedly decreased the overexpression of miR-196b/ miR-1290 and elevated IGFBP3 expression in the ALL cell lines. As an miR-196b/miR-1290 inhibitor, resveratrol was further demonstrated to exert antitumor effects on ALL cells including antiproliferation, cell cycle arrest, apoptosis and inhibition of migration. Dual-luciferase reporter assay revealed that $\mathrm{miR}-196 \mathrm{~b} / \mathrm{miR}-1290$ directly bound to the 3'-untranslated (3'-UTR) region of IGFBP3 mRNA. Moreover, we observed that IGFBP3 short interfering RNA reversed the antitumor activity of resveratrol against ALL cells. Taken together, the present study provides evidence that resveratrol targets miR196b and miR-1290 for its antitumor activity in T-ALL and B-ALL, respectively. The present study also confirms that
\end{abstract}

Correspondence to: Dr Ping Mao, Department of Hematology, Guangzhou First People's Hospital, Guangzhou Medical University, 1 Panfu Road, Guangzhou, Guangdong 510180, P.R. China E-mail: maoping@kindstar.com.cn

Key words: miR-196b, miR-1290, resveratrol, acute lymphoblastic leukemia, insulin-like growth factor binding protein 3 both miR-196b and miR-1290 target the IGFBP3 3'-UTR and are potential therapeutic targets for ALL.

\section{Introduction}

Acute lymphoblastic leukemia (ALL) is one of the most common childhood ( 0 -15 years of age) hematologic malignancies $(1,2)$. In recent years, with more and more in-depth studies of ALL, the survival rate of ALL patients has been significantly improved (3). Complete remission (CR) has been attained in more than $95 \%$ of cases and the 5-year event-free survival (EFS) has reached 63-83\% in pediatric ALL patients (4), while, the CR and 3-5-year EFS of adult ALL patients have reached $75-89 \%$ and $40 \%$, respectively (5). Nevertheless, numerous patients still suffer from the adverse events caused by conventional treatment and die from relapse (6). Therefore, a better understanding of the mechanism underlying ALL and development of new strategies for improving efficiency of ALL therapy are required. Emerging evidence indicates that insulin-like growth factor binding protein 3 (IGFBP3) is inversely associated with leukemia (7). Low IGFBP3 is related to the high-risk of events such as recurrence and decreased remission at the time of diagnosis (6), suggesting that the downregulation of expression of IGFBP3 plays an important role in the development of ALL.

MicroRNAs (miRNAs) are a family of endogenous, conserved, small non-coding RNAs (20-25 nucleotides in length). The complementary messenger RNAs (mRNAs) can be directly targeted on the 3'-untranslated regions (3'-UTRs) and suppressed by miRNAs in eukaryotes $(8,9)$. Altered expression of miRNAs participates in a variety of biological processes such as carcinogenesis, immunity, infection, endocrine homeostasis, differentiation and apoptosis $(10,11)$. By targeting complementary genes to control the expression of tumor-suppressor or oncogenic proteins, miRNAs are considered to play a significant role in the biology of cancers and to regulate cell proliferation, migration, invasion and apoptosis in cancers (12), thereby suggesting that a promising alternative novel approach for cancer treatment may be provided by miRNAs. It was reported that miR-196b is one of the most upregulated miRNAs in T-cell ALL (T-ALL) $(13,14)$. In addition, regardless of treatment protocol, miR-1290 is capable to 
serve as a new biomarker in childhood B-cell ALL (B-ALL) patients for outcome (3). However, the detailed regulatory mechanism of miR-196b or miR-1290 in ALL is still not well understood.

Numerous chemotherapeutic and chemopreventive compounds have been developed from natural sources and offer potential new alternatives to treat cancers (15). Resveratrol (3,5,40-trihydroxy-trans-stilbene), a natural polyphenol, is widely used in Traditional Chinese medicines (TCMs; such as Polygonum cuspidatum and Rheum officinale Baill.) and is found in peanuts, blueberries, cranberries, red wine and grape skin $(16,17)$. Accumulating research suggests that resveratrol has a number of important pharmacological properties such as antiproliferative, antioxidant, cardio-protective and anti-inflammatory activities (18-20). Resveratrol also displays anticancer activities by disturbing the three stages of carcinogenesis: initiation, promotion and progression (21). Previous studies have demonstrated that resveratrol inhibited the cell growth and induced apoptosis in several ALL cell lines, suggesting the anti-ALL effect of this agent (22-25). Nevertheless, the molecular mechanism of resveratrol-mediated anti-ALL activity has not been fully elucidated.

The present study aimed to ascertain whether miR-196b and miR-1290 serve as novel targets involved in the antitumor effect of resveratrol in ALL and to explore the probable common regulatory mechanism focusing on IGFBP3.

\section{Materials and methods}

Clinical samples. Peripheral blood and bone marrow samples were collected from 15 pairs of ALL patients and healthy volunteers at the Department of Hematology, Guangzhou First People's Hospital, Guangzhou Medical University, Guangzhou, Guangdong, China. Density gradient separation was used to isolate the human peripheral blood mononuclear cells (PBMCs) from whole blood by Ficoll-Paque Plus (GE Healthcare Bio-Sciences AB, Uppsala, Sweden) and the samples were then cryopreserved in liquid nitrogen with $90 \%$ fetal bovine serum (FBS) (Gibco, Carlsbad, CA, USA) and $10 \%$ dimethyl sulfoxide (DMSO) until analyzed.

Ethics statements. Permission to use the human bone marrow and peripheral blood samples for the present study was approved by the Ethics Committee of Guangzhou First People's Hospital (Guangdong, China).

Cell lines and cell culture. American Type Culture Collection (ATCC) (Manassas, VA, USA) provided the human embryonic kidney 293T, T-ALL TALL-104 and B-ALL SUP-B15 cells. 293T cells were cultured in Dulbecco's modified Eagle's medium (DMEM) with 10\% FBS and $2 \mathrm{mM} \mathrm{L}$-glutamine (Invitrogen Life Technologies, Carlsbad, CA, USA). TALL-104 cells were cultured in complete medium [ATCCformulated Iscove's modified Dulbecco's medium (IMDM) with $20 \%$ FBS, supplemented with $2.5 \mathrm{mg} / \mathrm{ml}$ human albumin, $0.5 \mathrm{mg} / \mathrm{ml} \mathrm{D-mannitol} \mathrm{and} 100 \mathrm{U}$ recombinant human IL-2 (all from Sigma-Aldrich, St. Louis, MO, USA)]. SUP-B15 cells were cultured in IMDM, supplemented with $10 \% \mathrm{FBS}, 2 \mathrm{mM}$ L-glutamine, $0.05 \mu \mathrm{M}$ 2- $\beta$-mercaptoethanol (Sigma-Aldrich),
$100 \mu \mathrm{g} / \mathrm{ml}$ streptomycin and $100 \mathrm{U} / \mathrm{ml}$ penicillin (Gibco). The cells were maintained at $37^{\circ} \mathrm{C}$ in a humidified atmosphere containing $5 \% \mathrm{CO}_{2}$.

Reagents. Resveratrol was obtained from Sigma-Aldrich. IGFBP3 siRNA and negative control siRNA were purchased from GenePharma (Shanghai, China). The antibody against IGFBP3, caspase-3 and GAPDH were purchased from Santa Cruz Biotechnology (Santa Cruz, CA, USA). The Cell Counting Kit-8 (CCK-8) was purchased from Dojindo Molecular Technologies (Kumamoto, Japan).

Cell proliferation assay. The cells were seeded into 96-well cell culture plates, and were incubated at $37^{\circ} \mathrm{C}$ for $0,24,48$ or $72 \mathrm{~h}$ in $5 \% \mathrm{CO}_{2}$. Cell proliferation was assessed via the CCK-8 assay. The numerical values obtained on an enzyme-labeled instrument (Thermo Fisher Scientific, Germany) with $450 \mathrm{~nm}$ wavelength were used to compare the cell viability.

Flow cytometry. Cells were collected, washed in ice-cold phosphate-buffered saline (PBS) and fixed in ice-cold $70 \%$ ethanol $\left(4^{\circ} \mathrm{C}\right.$, overnight). After centrifugation $(1,000 \mathrm{rpm}$, $5 \mathrm{~min}$ ), the cells were diluted with PBS and re-centrifuged. For the cell cycle assay, the cells were stained using a cell cycle kit (LiankeBio, Zhejiang, China) and incubated in the dark at $37^{\circ} \mathrm{C}$ for $30 \mathrm{~min}$. For analysis of apoptosis, the cells were stained using the Annexin V-FITC apoptosis detection kit (LiankeBio) and incubated in the dark at room temperature for $15 \mathrm{~min}$. Stained cells were detected via flow cytometry with a BD FACSCalibur (BD Biosciences, Heidelberg, Germany).

Cell migration assay. The migration of cells was performed in a Boyden Transwell chamber (Millipore, Bedford, MA, USA) containing a polycarbonate filter with a pore size of $8-\mu \mathrm{m}$. A cell suspension $(0.2 \mathrm{ml})\left(1 \times 10^{5}\right.$ cell $\left./ \mathrm{ml}\right)$ was added to the upper compartment of each chamber lined with an uncoated membrane. The bottom chamber was filled with $0.6 \mathrm{ml}$ IMDM containing $10 \%$ FBS as a chemoattractant. After incubation for $48 \mathrm{~h}$ at $37^{\circ} \mathrm{C}$ with $5 \% \mathrm{CO}_{2}$, the non-filtered cells were gently removed with a cotton swab and fixed with $4 \%$ paraformaldehyde. Filtered cells on the lower surface of the chamber were stained with $0.1 \%$ crystal violet (Sigma-Aldrich) and quantified manually in five random fields under a microscope (Olympus, Tokyo, Japan).

Quantitative real-time RT-PCR ( $q R T-P C R)$. Total RNA was extracted from the cells with TRIzol reagent (Invitrogen, Carlsbad, CA, USA) according to the manufacturer's instructions. The mRNA expression of IGFBP3, miR-196b or miR-1290 was detected by qRT-PCR using the standard SYBR-Green RT-PCR kit (Takara, Tokyo, Japan) following the manufacturer's manual. Real-time RT-PCR was performed using a sequence detector (Sigma-Aldrich). Specific primers were obtained from Genepharma: IGFBP3 forward, 5'-ATAA TCATCATCAAGAAAGGGCA-3' and reverse, 5'-AGTTCTG GGTATCTGTGCTCTGA-3'; miR-196b forward, 5'-ACAC TCCAGCTGGGTAGGTAGTTTCATG-3' and reverse, 5'-CT CAACTGGTGTCGTGGAGTCGGCAATTCAGTTGAGCC CAACAA-3'; miR-1290 forward, 5'-ACACTCCAGCTGGGT GGATTTTTGGATC-3' and reverse, 5'-CTCAACTGGTGTC 
GTGGAGTCGGCAATTCAGTTGAGTCCCTG-3'. The relative expression levels were calculated using the $2^{-\Delta \Delta \mathrm{Ct}}$ method.

Western blotting. Protein was extracted from the peripheral blood or cells using RIPA lysis buffer with a proteinase inhibitor. The protein concentration in the lysates was quantitated with the BCA Protein Assay kit (Bio-Rad, Hercules, CA, USA). Proteins were resolved on $10 \%$ SDS-PAGE gels under reducing conditions, followed by electrophoretic transfer onto polyvinylidene difluoride membranes (Millipore). Immunoblots were incubated with primary antibodies against IGFBP3 $(1: 2,000)$ or caspase-3 $(1: 1,000)$ (both from Abcam, Cambridge, USA) at $4^{\circ} \mathrm{C}$ overnight. Immunoreactive bands were detected using horseradish peroxidase (HRP)-conjugated secondary antibodies (1:20,000; Boster, Wuhan, China) with the Western Lightning Chemiluminescence Plus reagent (Perkin-Elmer Life Sciences, Boston, MA, USA). GAPDH was selected as the reference protein.

Dual-luciferase reporter assay. Cells were co-transfected with psiCHECK2-IGFBP3 3'-UTR or psiCHECK2-IGFBP3 3'-UTR mutant and miR-196b/miR-1290 mimics. Cells were lysed and the firefly luciferase activity was detected. Renilla luciferase activity was used for normalization. The lysate was detected using Dual-Luciferase Reporter Assay System (Promega, Madison, WI, USA) with a luminometer (Turner Designs, Sunnyvale, CA, USA).

Immunohistochemistry. Specimens were embedded in paraffin and a rotary microtome was used (HM355; Microm, Walldorf, Germany) to prepare serial sections with $3-\mu \mathrm{m}$ thickness. Some sections were stained with hematoxylin and eosin (H\&E) according to the manufacturer's protocol (SigmaAldrich). Before immunostaining, antigen retrieval was carried out via the treatment of $0.1 \%$ pepsin with $10 \mathrm{mM} \mathrm{HCl}$ at $37^{\circ} \mathrm{C}$ for $10 \mathrm{~min}$. The slides were incubated with the monoclonal mouse anti-human IGFBP3 (1:500; Sigma-Aldrich), and then anti-mouse IgG conjugated to HRP (Santa Cruz Biotechnology) for immunohistochemistry. The slides were exoposed to diaminobenzidine for $5 \mathrm{~min}$ and counterstained with hematoxylin (both from Sigma-Aldrich). A microscope (Olympus) was used to obtain the images.

Short interfering (si)RNA transfection. Synthetic IGFBP3 siRNA (20 ng) (Ambion, Austin, TX, USA) and the respective negative control were delivered into TALL-104 or SUP-B15 cells using Lipofectamine ${ }^{\mathrm{TM}}$ RNAiMAX (Life Technologies Corp., Carlsbad, CA, USA). Briefly, the cells were seeded into 6-well plates at $30 \%$ confluency. On the following day, IGFBP3 siRNA and the negative control were diluted in serum-free medium, and incubated with Lipofectamine ${ }^{\mathrm{TM}}$ RNAiMAX transfection reagent for $20 \mathrm{~min}$ at room temperature. The plates were gently swirled when adding the transfection complexes to the cell cultures. Fresh media were used to replace the culture media after $6 \mathrm{~h}$ and then the cells were incubated for $48 \mathrm{~h}$.

Statistical analysis. All data are expressed as the mean \pm SD. Student's t-test was used to evaluate the differences between two groups. For multiple comparisons, statistically significant

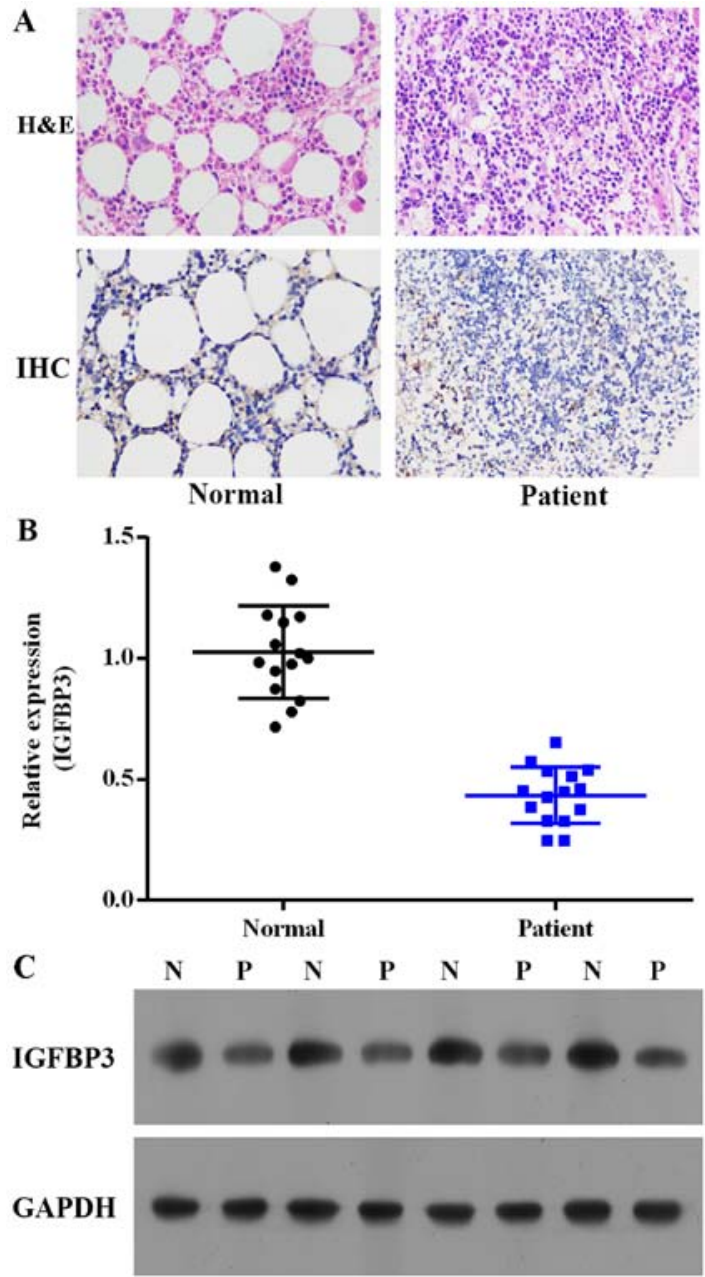

Figure 1. IGFBP3 expression is decreased in ALL patient samples. (A) Hematoxylin and eosin (H\&E) staining and immunohistochemistry (IHC) of bone marrow. The protein expression of IGFBP3 in samples from ALL patients (patient) was notably decreased compared with samples from the healthy volunteers (normal). (B) The mRNA expression levels of IGFBP3 in 15 pairs of peripheral blood samples from ALL patients and healthy volunteers were detected by qRT-PCR. (C) The protein expression of IGFBP3 was detected in ALL patients $(\mathrm{P})$ or the healthy volunteers $(\mathrm{N})$ by western blotting.

differences were assessed via one-way ANOVA. P-value $<0.05$ was considered to indicate a statistically significant.

\section{Results}

IGFBP3 expression is decreased in ALL patients. To explore the role of IGFBP3 in ALL, we initially examined the protein expression of IGFBP3 in 15 pairs of bone marrow from ALL patients and the healthy volunteers by immunohistochemistry. As depicted in Fig. 1A, the IGFBP3 expression in ALL patients was notably decreased compared with the level in the healthy volunteers. We further investigated the expression levels of IGFBP3 mRNA and protein in peripheral blood from the ALL patients and the healthy volunteers. As depicted in Fig. 1B and C, the mRNA and protein expression levels of IGFBP3 were decreased in the ALL patients compared with these levels in the healthy volunteers.

Resveratrol exerts an antitumor effect by the regulation of miR-196b/miR-1290 in ALL cells. Resveratrol 

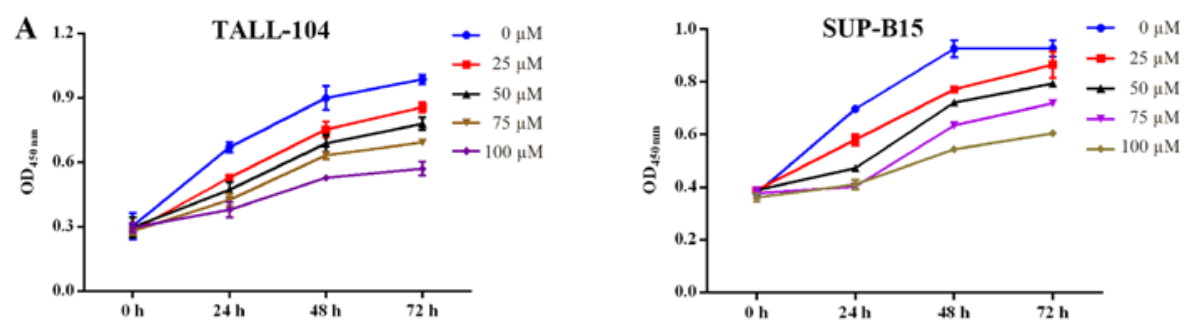

B
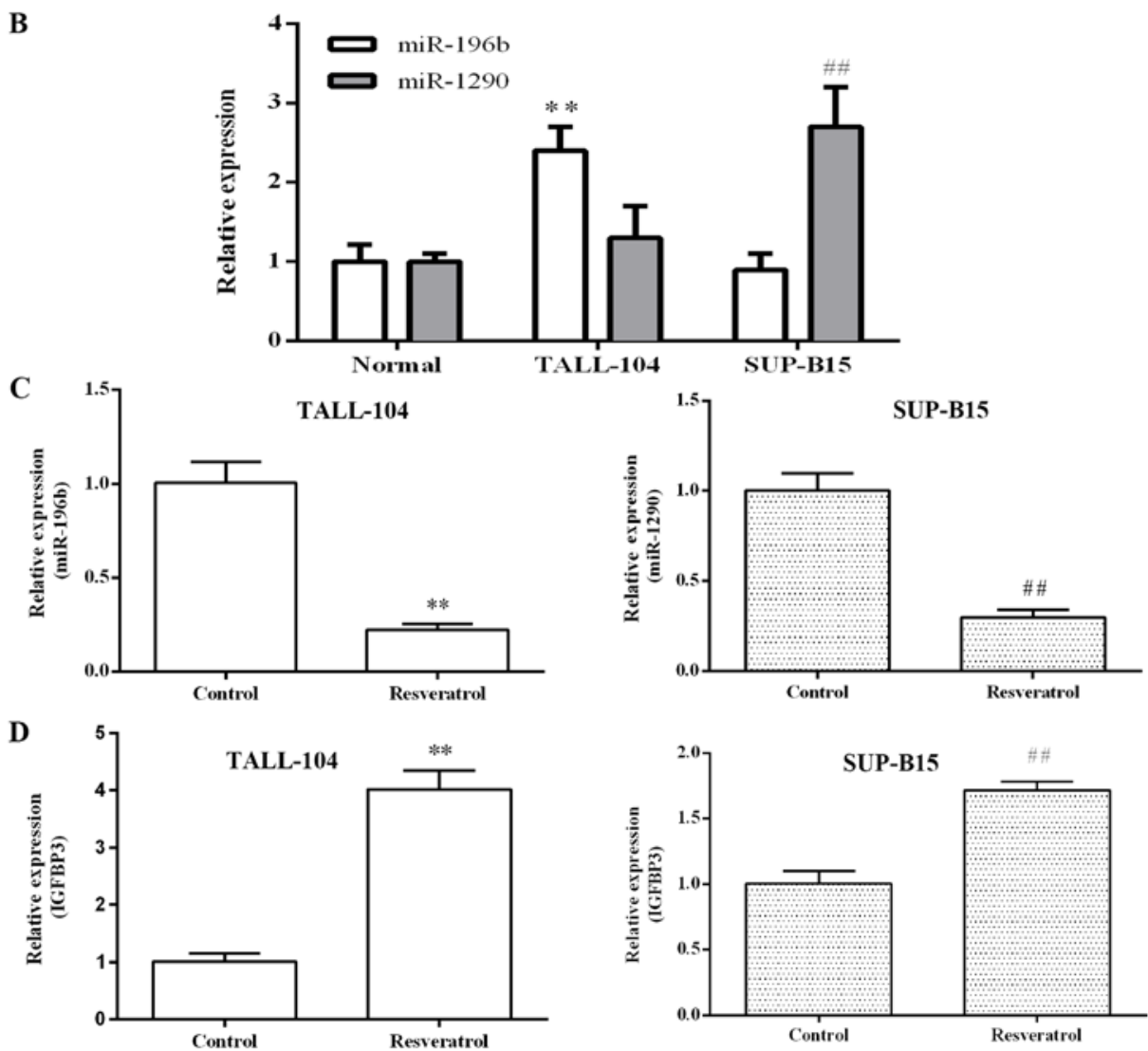

$\mathbf{E}$
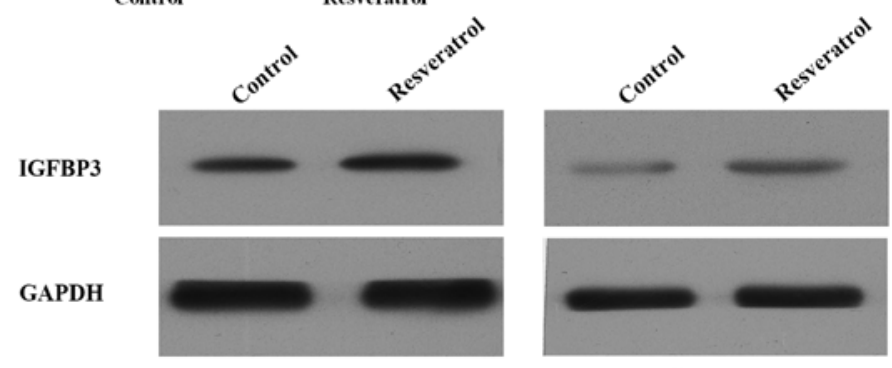

TALL-104

SUP-B15

Figure 2. Resveratrol exerts an antiproliferation effect by regulating miR-196b/miR-1290 in ALL cells. (A) Effect of resveratrol on the proliferation of TALL104 and SUP-B15 cells. Cells were incubated overnight, exposed to resveratrol $(25,50,75$ and $100 \mu \mathrm{M})$ and then cultured for 0, 24, 48 and $72 \mathrm{~h}$. Inhibition of proliferation was measured via the CCK-8 assay. (B) PBMCs (normal), TALL-104 or SUP-B15 cells were collected and the relative expression of miR-196b or miR-1290 was quantitated by qRT-PCR. (C) TALL-104 and SUP-B15 cells were treated with vehicle (control) or resveratrol at $75 \mu \mathrm{M}$ for $48 \mathrm{~h}$. Total RNA was extracted and the relative expression of miR-196b or miR-1290 was quantitated by qRT-PCR. (D) The mRNA expression of IGFBP3 in TALL-104 or SUP-B15 cells after the treatment of vehicle (control) or $75 \mu \mathrm{M}$ resveratrol for $48 \mathrm{~h}$. (E) The protein expression of IGFBP3 in TALL-104 or SUP-B15 cells after the treatment of vehicle (control) or $75 \mu \mathrm{M}$ resveratrol for $48 \mathrm{~h}$ was detected by western blotting. The results are expressed as the mean $\pm \mathrm{SD}$ of three independent experiments; ${ }^{*} \mathrm{P}<0.01$ and ${ }^{\# \#} \mathrm{P}<0.01$, statistically significant difference from the normal/control group.

dose- and time-dependently inhibited the proliferation of TALL-104 and SUP-B15 cells (Fig. 2A). Previous miRNA microarray profiling indicated that miR-196b was upregulated in T-ALL and miR-1290 was upregulated in B-ALL. As shown in Fig. 2B, we confirmed that the miR-196b expression level was significantly increased in TALL-104 cells compared with the level in the PBMCs $(\mathrm{P}<0.01)$, and miR-1290 was overexpressed in the SUP-B15 cells $(\mathrm{P}<0.01)$. qRT-PCR was performed to investigate whether resveratrol regulates miR196b/miR-1290 in ALL cells. As shown in Fig. 2C, resveratrol markedly inhibited miR-196b/miR-1290 expression in TALL104/SUP-B15 cells, respectively. Furthermore, we found that resveratrol elevated IGFBP3 mRNA and protein expression in both TALL-104 and SUP-B15 cells (Fig. 2D and E). 

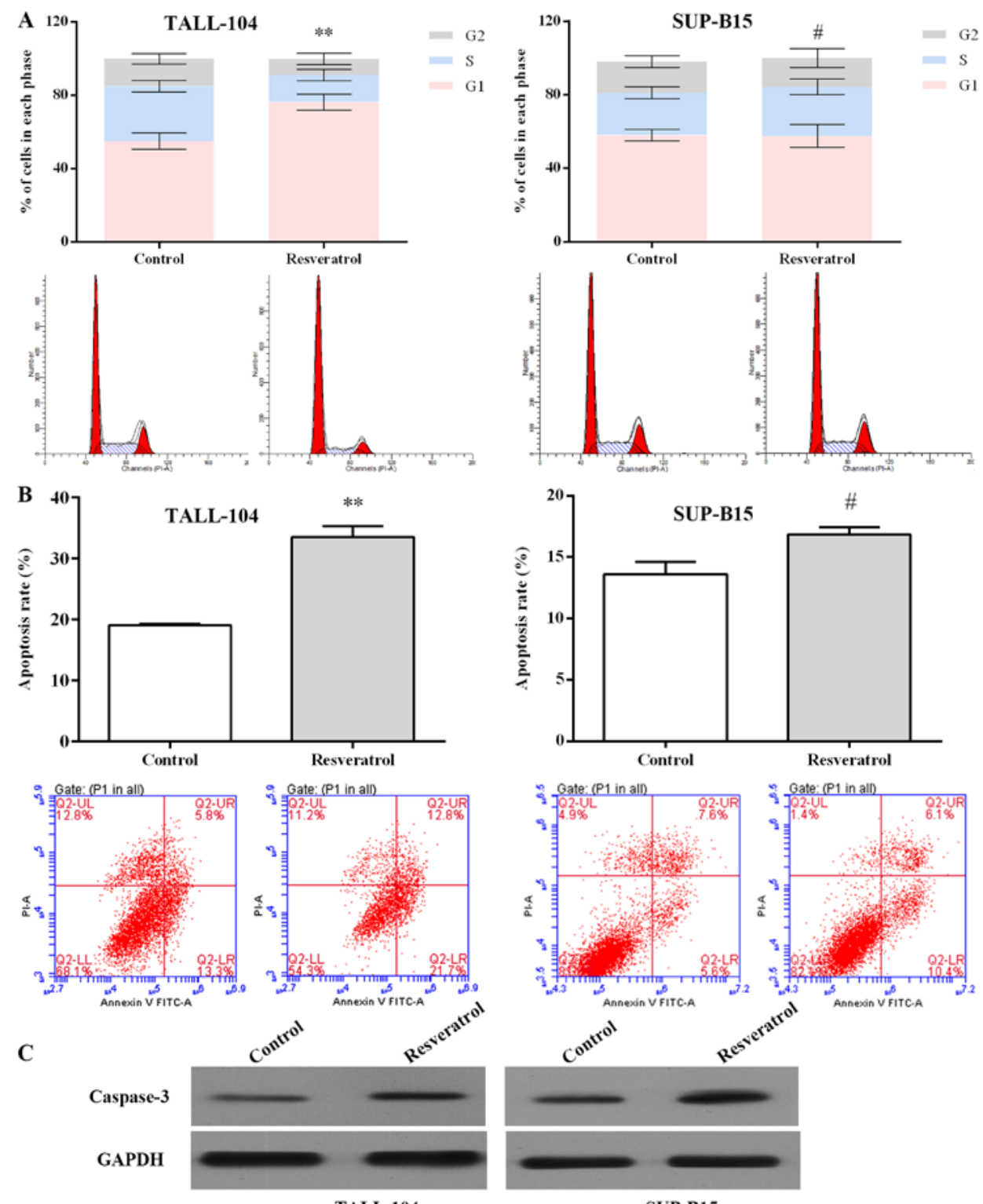

Figure 3. Resveratrol induces cell cycle arrest and apoptosis in ALL cells. Cells were treated with vehicle (control) or $75 \mu \mathrm{M}$ resveratrol for $48 \mathrm{~h}$. (A) Resveratrol treatment caused cell cycle arrest at the G1 phase in TALL-104 cells and S phase in SUP-B15 cells, as analyzed for propidium iodide stained-DNA content via flow cytometry. (B) Resveratrol treatment significantly increased the apoptosis of both TALL-104 and SUP-B15 cells, as determined by Annexin V-FITC staining using flow cytometry. (C) Resveratrol elevated the protein expression of caspase-3 in both TALL-104 and SUP-B15 cells, as detected by western blotting. Data in the bar graphs are expressed as mean $\pm \mathrm{SD} ; \mathrm{n}=3 ;{ }^{* *} \mathrm{P}<0.01,{ }^{,} \mathrm{P}<0.05$.
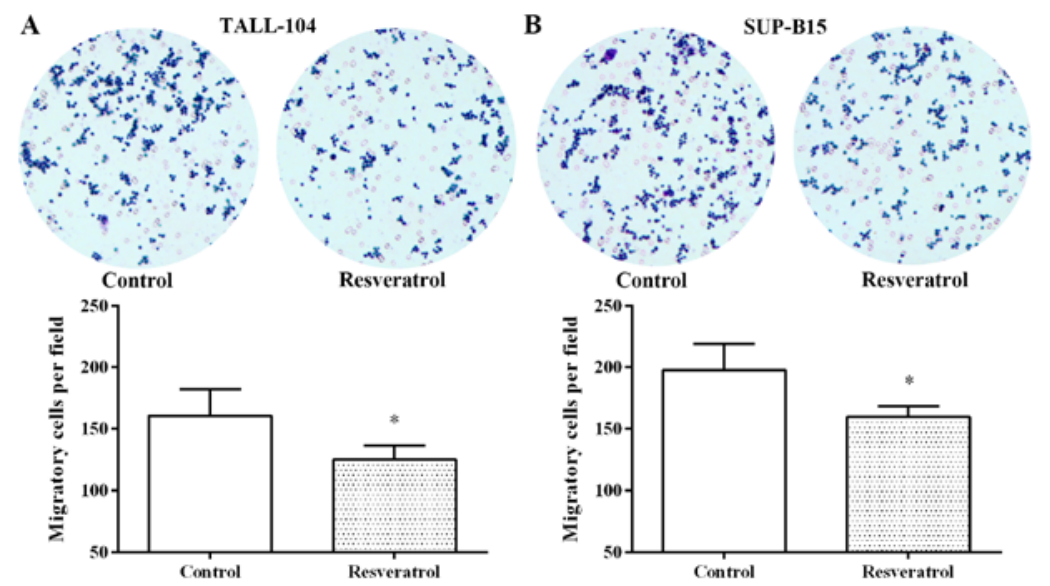

Figure 4. Resveratrol suppresses the migration capability of ALL cells. Cells were treated with vehicle (control) or $75 \mu \mathrm{M}$ resveratrol for $48 \mathrm{~h}$. Resveratrol notably inhibited cell migration in both the (A) TALL-104 and (B) SUP-B15 cells, as examined by Transwell assay. Data in the bar graphs are expressed as mean $\pm \mathrm{SD} ; \mathrm{n}=3 ;{ }^{*} \mathrm{P}<0.05$. 
A

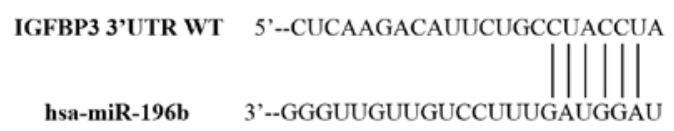

IGFBP3 3'UTR MUT 5'--CUCAAGACAUUCUGCGAUGGAA

B

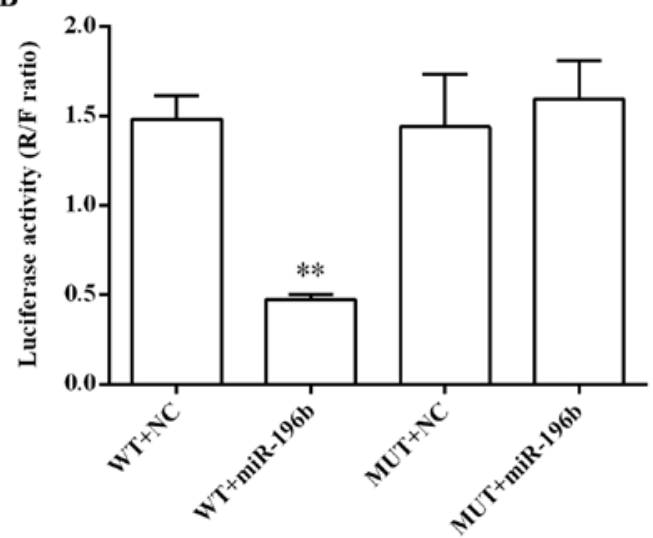

IGFBP3 3'UTR WT 5'--UUUAUUAUAAUAAAAUCCU

hsa-miR-1290

3'--AGGGACUAGGUUUUUAGGU

Figure 5. miR-196b and miR-1290 directly target IGFBP3 in ALL cells. (A) The putative binding sites of miR-196b and miR-1290 within the IGFBP3 3'-UTR WT (wild-type) are indicated. (B) Relative luciferase activity of the indicated IGFBP3 reporter constructs. The luciferase activity was suppressed in the cells co-transfected with IGFBP3-WT and miR-196b/miR-1290. Each value represents the mean \pm SD; $n=3$. $\left(^{* *}\right)$ and $\left(^{\# \#)}\right.$ indicate statistically significant difference $(\mathrm{P}<0.01)$ from the WT + NC (IGFBP3-WT $\left.{ }^{+}, \mathrm{miR}-196 \mathrm{~b} / \mathrm{miR}-1290\right)$ group.
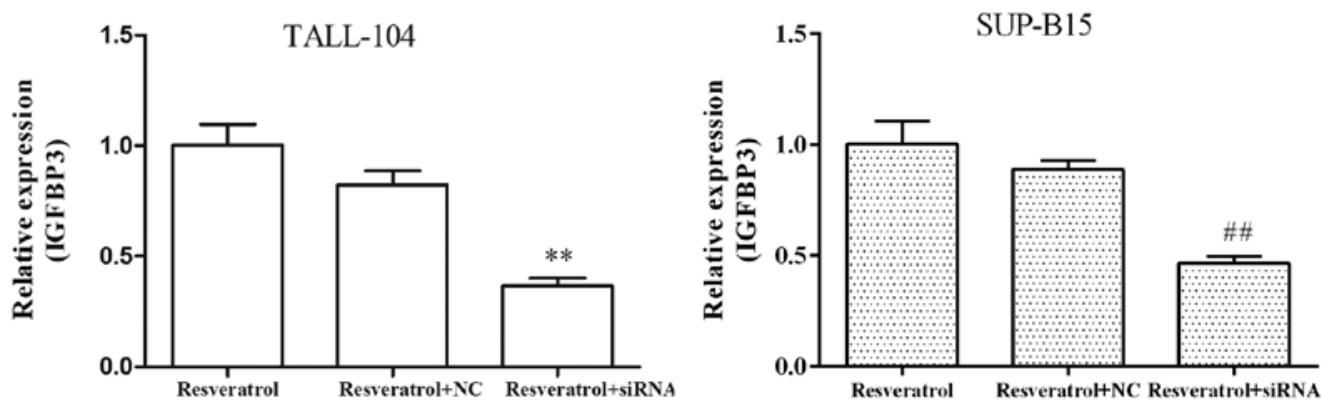

Figure 6. IGFBP3 siRNA reduces the upregulation of IGFBP3 expression by resveratrol. TALL-104 and SUP-B15 cells were transfected with either scramble (NC) or IGFBP3 siRNA and then exposed to $75 \mu \mathrm{M}$ resveratrol for $48 \mathrm{~h}$. mRNA expression level of IGFBP3 was detected by qRT-PCR. Each point represents the mean $\pm \mathrm{SD} ; \mathrm{n}=3 ;{ }^{*} \mathrm{P}<0.01$ and ${ }^{\# \#} \mathrm{P}<0.01$, statistically significant difference from the resveratrol $+\mathrm{NC}$ group.

As an miR-196b/miR-1290 inhibitor, resveratrol was further examined in regards to its antitumor effect. As displayed in Fig. 3A, resveratrol arrested the cell cycle at the G1 phase in TALL-104 cells $(\mathrm{P}<0.01)$, and arrested the cell cycle at $\mathrm{S}$ phase in SUP-B15 cells $(\mathrm{P}<0.05)$. Resveratrol increased the apoptotic rate in the TALL-104 and SUP-B15 cells notably when compared with the rate in the control group (Fig. 3B). Activation of caspase-3 is significant in apoptosis (26). As shown in Fig. 3C, resveratrol markedly upregulated the caspase- 3 expression in both TALL-104 and SUP-B15 cells. Furthermore, resveratrol also notably inhibited cell migration in the TALL-104 and SUP-B15 cells (Fig. 4). These findings suggest that resveratrol exerted an anti-ALL effect by regulating miR-196b/miR-1290.

Both miR-196b and miR-1290 target IGFBP3 in ALL cells. As shown in Fig. 5A, the predicted binding sites of miR-196b and miR-1290 within the $3^{\prime}$-UTR of the IGFBP3 gene are indicated. To confirm their relationship, we further performed dual-luciferase reporter assay. As shown in Fig. 5B, the relative luciferase activity was markedly decreased after co-transfection with the wild-type 3'-UTR of IGFBP3 and miR-196b or miR-1290 in 293T cells ( $\mathrm{P}<0.01$, respectively), while the mutant 3'-UTR of IGFBP3 showed slight inhibitory function on the luciferase activity, suggesting that both miR-196b and miR-1290 suppressed the transcription activity of the IGFBP3 gene by directly targeting the binding site in the 3'-UTR of IGFBP3 mRNA.

siRNA against IGFBP3 attenuates the antitumor effect of resveratrol on ALL cells. Resveratrol markedly upregulated the expression levels of IGFBP3 in both TALL-104 and SUP-B15 cells (Fig. 2D and E), indicating that resveratrol exhibited a common response in the different types of ALL 

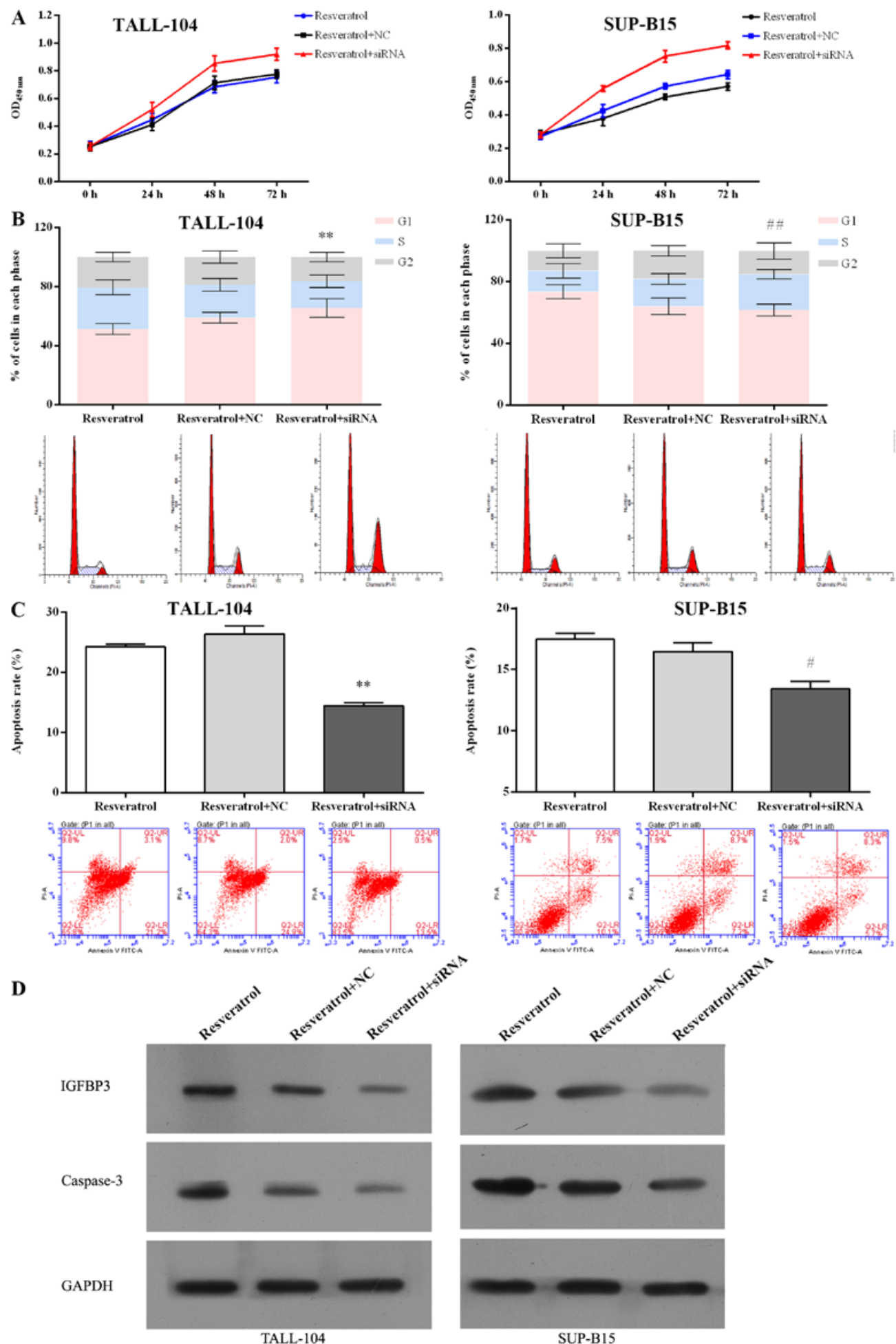

Figure 7. Downregulation of IGFBP3 attenuates the antitumor effect of resveratrol in ALL cells. TALL-104 and SUP-B15 cells were transfected with either scramble (NC) or IGFBP3 siRNA. (A) Cells were exposed to $75 \mu \mathrm{M}$ resveratrol for $0,24,48$ or $72 \mathrm{~h}$. Cell viability was evaluated via the CCK-8 assay. (B) Cells were exposed to $75 \mu \mathrm{M}$ resveratrol for $48 \mathrm{~h}$. The cell cycle progression was analyzed for propidium iodide stained-DNA content via flow cytometry. (C) Cell apoptosis assay was carried out by Annexin V-FITC staining using flow cytometry. (D) The protein expression of IGFBP3 and caspase-3 was detected by western blotting. Data in the bar graphs are expressed as mean $\pm \mathrm{SD} ; \mathrm{n}=3 ;{ }^{* *} \mathrm{P}<0.01,{ }^{\# \#} \mathrm{P}<0.01,{ }^{\#} \mathrm{P}<0.05$ vs. the resveratrol $+\mathrm{NC}$ group.

cell lines. To determine the role of miR-196b/miR-1290 in the antitumor efficacy of resveratrol against ALL, we first transfected both TALL-104 and SUP-B15 cells with IGFBP3 siRNA, and then examined whether IGFBP3 siRNA affects the antitumor actions of resveratrol in ALL cells. As shown in Fig. 6, targeting IGFBP3 by siRNA resulted in marked attenuation of the absolute induction of mRNA expression levels of IGFBP3 observed following treatment of resveratrol. Furthermore, IGFBP3 siRNA blocked the inhibitory effect pf proliferation mediated by resveratrol (Fig. 7A) in both TALL-104 and SUP-B15 cells. IGFBP3 siRNA also attenuated the ability of resveratrol to induce cell cycle arrest (Fig. 7B) 
and cell apoptosis (Fig. 7C and D) in both TALL-104 and SUP-B15 cells. These data indicate that downregulation of IGFBP3 attenuated the anti-ALL effect of resveratrol, suggesting that $\mathrm{miR}-196 \mathrm{~b} / \mathrm{miR}-1290$ play a pivotal role in the antitumor effect of resveratrol in ALL cells.

\section{Discussion}

Accumulating evidence suggests that miRNAs may function as oncogenes or tumor suppressors in human cancer development $(27,28)$. In acute lymphoblastic leukemia (ALL), different miRNAs have been reported to play critical roles in T-ALL and B-ALL (29). For example, miRNA-193b-3p was reported to be a potential tumor-suppressor in T-ALL (30) and miRNA-17-92 was found to play a critical role in B-ALL (31). In the present study, we identified that miR-196b and miR-1290 were overexpressed in T-ALL and B-ALL cells, respectively. However, the function of the two cellular miRNAs in ALL and their potential contribution to ALL therapy are still not well clarified.

It is well known that miRNAs function by regulating the expression of complementary genes. We hypothesized whether there is a key target co-regulated by the different miRNAs in T-ALL and B-ALL. We found various studies concerning IGFBP3, which is downregulated and acts as a key target in $\operatorname{ALL}(7,32)$. In the present study, we validated that the expression of IGFBP3 was decreased in both bone marrow and peripheral blood of the 15 ALL patients, which was in accordance with previous studies. Then, we further explored whether IGFBP3 can be co-regulated by different miRNAs and its role in T-ALL and B-ALL. The results indicate that both miR-196b and miR-1290 directly bind to the 3'-UTR of IGFBP3, suggesting the negative regulation of IGFBP3 expression in T-ALL and B-ALL cells by miR-196b and miR-1290, respectively.

Resveratrol has been reported to possess antitumor effects via regulation of specific miRNAs and alteration of the crucial gene expression they target in colorectal (33), pancreatic (27) and bladder cancer (34), and glioma (15). However, the regulation of miRNAs by resveratrol in ALL warrants further investigation. We initially used two ALL cell lines: T-ALL TALL-104 and B-ALL SUP-B15 to examine the potential antiproliferation effect of resveratrol. Resveratrol exhibited similar antiproliferative activities in TALL-104 and SUP-B15 cells. Moreover, resveratrol markedly decreased the overexpression of miR-196b and miR-1290. Numerous published studies in recent years have demonstrated the fundamental roles of miRNAs in carcinogenesis, cell proliferation, migration, invasion and apoptosis (35). As an miR-196b/miR-1290 inhibitor, resveratrol was further found to induce cell cycle arrest, apoptosis, and inhibit migration in ALL cells. The data suggest that miR-196b and miR-1290 may participate in the anti-ALL effect of resveratrol, which needs more confirmation.

By applying IGFBP3 siRNA, we found that knockdown of IGFBP3 reversed the antiproliferation, cell cycle arrest, apoptosis induction abilities of resveratrol in both T-ALL and B-ALL cells. According to the pieces of evidence, we conclude that resveratrol exhibits anticancer activity in T-ALL and B-ALL by targeting miR-196b and miR-1290, respectively. However, it should be noted that each miRNA targets a diversity of genes.
Li et al reported that miR-196b directly targets both FAS tumorsuppressor and HOXA9/MEIS1 oncogenes in MLL-rearranged leukemia (36). Endo et al revealed that miR-1290 decreased the expression of forkhead box A1 and N-acetyltransferase-1 in ER-positive breast cancer (37). The complex functions of miR-196b $(36,38)$ and miR-1290 $(37,39)$ in tumors indicate that resveratrol may exert antitumor activity against other cancers as an miR-196b/miR-1290 inhibitor and the modulating mechanism of miR-196b/miR-1290 in ALL warrants further exploration.

In summary, miR-196b and miR-1290 were identified as new targets of resveratrol. miR-196b and miR-1290 mediated the inhibition of T-ALL and B-ALL cell growth, survival and migration achieved by resveratrol. The findings also support that both miR-196b and miR-1290 target the IGFBP3 3'-UTR and may be potential therapeutic targets for ALL.

\section{Acknowledgements}

This study was supported by grants from the National Natural Science Foundation of China (no. 81600147) and the Health Collaborative Innovation Major Projects of Guangzhou City (201508020254).

\section{References}

1. Inaba H, Greaves M and Mullighan CG: Acute lymphoblastic leukaemia. Lancet 381: 1943-1955, 2013.

2. Luan C, Yang $Z$ and Chen B: The functional role of microRNA in acute lymphoblastic leukemia: Relevance for diagnosis, differential diagnosis, prognosis, and therapy. Onco Targets Ther 8: 2903-2914, 2015 .

3. Avigad S, Verly IR, Lebel A, Kordi O, Shichrur K, Ohali A, Hameiri-Grossman M, Kaspers GJ, Cloos J, Fronkova E, et al: $\mathrm{miR}$ expression profiling at diagnosis predicts relapse in pediatric precursor B-cell acute lymphoblastic leukemia. Genes Chromosomes Cancer 55: 328-339, 2016.

4. Pui $\mathrm{CH}$ and Evans WE: Treatment of acute lymphoblastic leukemia. N Engl J Med 354: 166-178, 2006.

5. Adil SN and Usman M: Adult acute lymphoblastic leukemia. J Pak Med Assoc 54: 444, 2004.

6. Vorwerk P, Mohnike K, Wex H, Röhl FW, Zimmermann M, Blum WF and Mittler U: Insulin-like growth factor binding protein-2 at diagnosis of childhood acute lymphoblastic leukemia and the prediction of relapse risk. J Clin Endocrinol Metab 90: 3022-3027, 2005.

7. Petridou E, Dessypris N, Spanos E, Mantzoros C, Skalkidou A, Kalmanti M, Koliouskas D, Kosmidis H, Panagiotou JP, Piperopoulou F, et al: Insulin-like growth factor-I and binding protein-3 in relation to childhood leukaemia. Int $\mathrm{J}$ Cancer 80: 494-496, 1999.

8. Inui M, Martello G and Piccolo S: MicroRNA control of signal transduction. Nat Rev Mol Cell Biol 11: 252-263, 2010.

9. Bartel DP: MicroRNAs: Target recognition and regulatory functions. Cell 136: 215-233, 2009.

10. Bartel DP: MicroRNAs: Genomics, biogenesis, mechanism, and function. Cell 116: 281-297, 2004.

11. Leal JA and Lleonart ME: MicroRNAs and cancer stem cells: Therapeutic approaches and future perspectives. Cancer Lett 338: 174-183, 2013.

12. Yi B, Piazza GA, Su X and Xi Y: MicroRNA and cancer chemoprevention. Cancer Prev Res 6: 401-409, 2013.

13. Ghisi M, Corradin A, Basso K, Frasson C, Serafin V, Mukherjee S, Mussolin L, Ruggero K, Bonanno L, Guffanti A, et al: Modulation of microRNA expression in human T-cell development: Targeting of NOTCH3 by miR-150. Blood 117: 7053-7062, 2011.

14. Cammarata G, Augugliaro L, Salemi D, Agueli C, La Rosa M, Dagnino L, Civiletto G, Messana F, Marfia A, Bica MG, et al: Differential expression of specific microRNA and their targets in acute myeloid leukemia. Am J Hematol 85: 331-339, 2010. 
15. Wang G, Dai F, Yu K, Jia Z, Zhang A, Huang Q, Kang C, Jiang H and $\mathrm{Pu} P$ : Resveratrol inhibits glioma cell growth via targeting oncogenic microRNAs and multiple signaling pathways. Int $\mathbf{J}$ Oncol 46: 1739-1747, 2015.

16. Su YC, Li SC, Wu YC, Wang LM, Chao KS and Liao HF: Resveratrol downregulates interleukin-6-stimulated sonic hedgehog signaling in human acute myeloid leukemia. Evid Based Complement Alternat Med 2013: 547430, 2013.

17. Chen BY, Kuo CH, Liu YC, Ye LY, Chen JH and Shieh CJ: Ultrasonic-assisted extraction of the botanical dietary supplement resveratrol and other constituents of Polygonum cuspidatum. J Nat Prod 75: 1810-1813, 2012.

18. Wang ZH, Zhang JL, Duan YL, Zhang QS, Li GF and Zheng DL: MicroRNA-214 participates in the neuroprotective effect of resveratrol via inhibiting $\alpha$-synuclein expression in MPTP-induced Parkinson's disease mouse. Biomed Pharmacother 74: 252-256, 2015.

19. Cullberg KB, Foldager CB, Lind M, Richelsen B and Pedersen SB: Inhibitory effects of resveratrol on hypoxia-induced inflammation in 3T3-L1 adipocytes and macrophages. J Funct Foods 7: 171-179, 2014.

20. Frémont L: Biological effects of resveratrol. Life Sci 66: 663-673, 2000.

21. Jang M, Cai L, Udeani GO, Slowing KV, Thomas CF, Beecher CW, Fong HH, Farnsworth NR, Kinghorn AD, Mehta RG, et al: Cancer chemopreventive activity of resveratrol, a natural product derived from grapes. Science 275: 218-220, 1997.

22. Ferry-Dumazet H, Garnier O, Mamani-Matsuda M, Vercauteren J, Belloc F, Billiard C, Dupouy M, Thiolat D, Kolb JP, Marit G, et al: Resveratrol inhibits the growth and induces the apoptosis of both normal and leukemic hematopoietic cells. Carcinogenesis 23: 1327-1333, 2002.

23. Azimi A, Hagh MF, Talebi M, Yousefi B, Hossein pour feizi AA, Baradaran B, Movassaghpour AA, Shamsasenjan K, Khanzedeh T, Ghaderi AH, et al: Time- and concentrationdependent effects of resveratrol on miR 15a and miR16-1 expression and apoptosis in the CCRF-CEM acute lymphoblastic leukemia cell line. Asian Pac J Cancer Prev 16: 6463-6468, 2015.

24. Ge J, Liu Y, Li Q, Guo X, Gu L, Ma ZG and Zhu YP: Resveratrol induces apoptosis and autophagy in T-cell acute lymphoblastic leukemia cells by inhibiting Akt/mTOR and activating p38-MAPK. Biomed Environ Sci 26: 902-911, 2013.

25. Ghorbani A, Zand H, Jeddi-Tehrani M, Koohdani F, Shidfar F and Keshavarz SA: PTEN over-expression by resveratrol in acute lymphoblastic leukemia cells along with suppression of AKT/PKB and ERK1/2 in genotoxic stress. J Nat Med 69: 507-512, 2015.

26. Riedl SJ and Shi Y: Molecular mechanisms of caspase regulation during apoptosis. Nat Rev Mol Cell Biol 5: 897-907, 2004.

27. Liu P, Liang H, Xia Q, Li P, Kong H, Lei P, Wang S and Tu Z: Resveratrol induces apoptosis of pancreatic cancers cells by inhibiting miR-21 regulation of BCL-2 expression. Clin Transl Oncol 15: 741-746, 2013.
28. Adams BD, Kasinski AL and Slack FJ: Aberrant regulation and function of microRNAs in cancer. Curr Biol 24: R762-R776, 2014.

29. Schotte D, Chau JC, Sylvester G, Liu G, Chen C, van der Velden VH, Broekhuis MJ, Peters TC, Pieters R and den Boer ML: Identification of new microRNA genes and aberrant microRNA profiles in childhood acute lymphoblastic leukemia. Leukemia 23: 313-322, 2009.

30. Mets E, Van der Meulen J, Van Peer G, Boice M, Mestdagh P, Van de Walle I, Lammens T, Goossens S, De Moerloose B, Benoit Y, et al: MicroRNA-193b-3p acts as a tumor suppressor by targeting the MYB oncogene in T-cell acute lymphoblastic leukemia. Leukemia 29: 798-806, 2015.

31. Scherr M, Elder A, Battmer K, Barzan D, Bomken S, Ricke-Hoch M, Schröder A, Venturini L, Blair HJ, Vormoor J, et al: Differential expression of miR-17 92 identifies BCL2 as a therapeutic target in BCR-ABL-positive B-lineage acute lymphoblastic leukemia. Leukemia 28: 554-565, 2014.

32. Mohnike KL, Kluba U, Mittler U, Aumann V, Vorwerk P and Blum WF: Serum levels of insulin-like growth factor-I, -II and insulin-like growth factor binding proteins -2 and -3 in children with acute lymphoblastic leukaemia. Eur J Pediatr 155: 81-86, 1996.

33. Yang S, Li W, Sun H, Wu B, Ji F, Sun T, Chang H, Shen P, Wang Y and Zhou D: Resveratrol elicits anti-colorectal cancer effect by activating $m i R-34 c$-KITLG in vitro and in vivo. BMC Cancer 15: 969, 2015

34. Zhou C, Ding J and Wu Y: Resveratrol induces apoptosis of bladder cancer cells via miR-21 regulation of the Akt/Bcl-2 signaling pathway. Mol Med Rep 9: 1467-1473, 2014.

35. Phuah NH and Nagoor NH: Regulation of microRNAs by natural agents: New strategies in cancer therapies. BioMed Res Int 2014: $804510,2014$.

36. Li Z, Huang H, Chen P, He M, Li Y, Arnovitz S, Jiang X, He C, Hyjek E, Zhang J, et al: miR-196b directly targets both HOXA9/MEIS1 oncogenes and FAS tumour suppressor in MLL-rearranged leukaemia. Nat Commun 3: 688, 2012.

37. Endo Y, Toy am a T, Takahashi S, Yoshimoto N, Iwasa M, Asano T, Fujii Y and Yamashita H: miR-1290 and its potential targets are associated with characteristics of estrogen receptor $\alpha$-positive breast cancer. Endocr Relat Cancer 20: 91-102, 2013.

38. Popovic R, Riesbeck LE, Velu CS, Chaubey A, Zhang J, Achille NJ, Erfurth FE, Eaton K, Lu J, Grimes HL, et al: Regulation of mir-196b by MLL and its overexpression by MLL fusions contributes to immortalization. Blood 113: 3314-3322, 2009.

39. Wu J, Ji X, Zhu L, Jiang Q, Wen Z, Xu S, Shao W, Cai J, Du Q, Zhu Y, et al: Up-regulation of microRNA-1290 impairs cytokinesis and affects the reprogramming of colon cancer cells. Cancer Lett 329: 155-163, 2013. 